\title{
THE BATTLE IN ENDODONTICS: A REVIEW
}

\author{
Artinder Kaur ${ }^{1}$, Devendra Chaudhary ${ }^{2}$, Navneet Kukreja ${ }^{3}$, Abhishek Bansal', Jyoti Bansal ${ }^{5}$, Urvashi Kukreja ${ }^{6}$ \\ ${ }^{1}$ Post Graduate Student, Department of Conservative Dentistry and Endodontics, MM College of Dental Sciences And Research, \\ Mullana, Ambala. \\ ${ }^{2}$ Prof. and Hod, Department of Conservative Dentistry and Endodontics, Vice Principal and Director PG studies, Maharaja Ganga Singh \\ Dental College Ganganagar, Rajasthan \\ ${ }^{3}$ Professor, Department of Conservative Dentistry and Endodontics, MM College of Dental Sciences and Research, Mullana, Ambala. \\ ${ }^{4}$ Associate Professor, Department of Conservative Dentistry And Endodontics, MM College of Dental Sciences and Research, \\ Mullana, Ambala. \\ ${ }^{5}$ Reader, Department of Periodontics, MM College of Dental Sciences and Research, Mullana, Ambala. \\ ${ }^{6}$ Reader, Department of Prosthodontics, MM College of Dental Sciences and Research, Mullana, Ambala.
}

Corresponding Author: Artinder Kaur Mobile: +91- 9872776653 E-mail: artindermavy@gmail.com

Received: $23^{\text {rd }}$ March 2014 Accepted: $19^{\text {th }}$ Aug 2014 Online: $20^{\text {th }}$ Sept 2014

\section{ABSTRACT}

Nickel titanium rotary shaping files fundamentally changed everything in endodontics; conceptually, procedurally and economically as well. NiTi rotary files made root canal therapy more accessible and kind of fun for dentists, and dental companies were loved for making files that cost four times more than stainless steel hand files but delivered a ten times improvement. The single file systems need no disinfecting, cleaning, sterilizing and organizing the NiTi files, provide optimal cutting efficiency along with better control of file breakage and thus increased patient safety and no risk for cross contamination.

Key words: Bio-Mechanical Preparation, Canal Centering Ability, Single File Rotary System

\section{Introduction}

Traditionally, the shaping of root canals was achieved by the use of stainless steel hand files. However, techniques using stainless steel hand files have several drawbacks. ${ }^{1}$ They require the use of numerous hand files and drills to adequately prepare the canals (Schilder 1947).Hand instrumentation with stainless steel files is time consuming (Ferraz et al. 2001).The stainless steel hand instrumentation techniques have an increased incidence of canal transportation (Kuhn et al. 1997, Reddy and Hicks 1998, Ferraz et al. 2001, Pettiette et al. 2001).Finally, from a clinical standpoint, the use of hand instruments in narrow canals can be very frustrating especially in teeth with difficult access. Nickel titanium rotary shaping files fundamentally changed everything in endodontics; conceptually, procedurally and economically as well. NiTi rotary files made root canal therapy more accessible and kind of fun for dentists, and dental companies were loved for making files that cost four times more than SS hand files but delivered a ten times improvement. ${ }^{1}$ Recent advances for endodontic canal preparation have focused on the concept "Less is More". Thus a single-file technique has developed for shaping the vast majority of canals, regardless of their length, diameter, or curvature. ${ }^{1}$

Today, there is a battle in single file systems too, as many single file system are now in market. Single file system which are there in market: Waveone, Reciproc, Oneshape and Komet f360.

\section{Wave One (DENTSPLY Maillefer):}

The new Wave One NiTi file system from DENTSPLY Maillefer is a single-use, single-file system to shape the root canal completely from start to finish. Shaping the root canal to a continuously tapering funnel shape not only fulfils the biological requirements for adequate irrigation to rid the root canal system of all bacteria, bacterial by-products and pulp tissue, ${ }^{2}$ but also provides the perfect shape for 3-D obturation with gutta-percha. ${ }^{3,4}$ In most 
cases, the technique only requires one hand file followed by one single Wave One file to shape the canal completely. The specially designed NiTi files work in a similar but reverse "balanced force" action using a preprogrammed motor to move the files in a back and forth "reciprocal motion". ${ }^{5}$ The files are manufactured using M-Wire technology, improving strength and resistance to cyclic fatigue by up to nearly four times in comparison with other brands of rotary NiTi files. ${ }^{6}$

There are many dentists who, for whatever reason, are reluctant to use NiTi rotary instruments to prepare canals, despite the recognized advantages of flexibility, less debris extrusion and maintaining canal shape, amongst other advantages. ${ }^{7}$ For them, the use of a single reciprocating file will be very attractive both in terms of time and cost saving. At present, there are three files in the Wave One single-file reciprocating system available in lengths of 21, 25 and $31 \mathrm{~mm}$ (Figure 1).

1. The Wave One Small file is used in fine canals. The tip size is ISO 21 with a continuous taper of $6 \%$.

2. The Wave One Primary file is used in the majority of canals. The tip size is ISO 25 with an apical taper of $8 \%$ that reduces towards the coronal end.

3. The WaveOne Large file is used in large canals. The tip size is ISO 40 with an apical taper of $8 \%$ that reduces towards the coronal end.

Speed: $300 \mathrm{rpm}$

Torque: $5 \mathrm{Ncm}$

The instruments are designed to work with a reverse cutting action. All instruments have a modified convex triangular cross-section (Figure 2) at the tip end, reciprocating cycles complete one complete reverse rotation and the instrument gradually advances into the canal with little apical pressure required. All brands of NiTi files can be used with the Wave One motor, as it has additional functions for continuous rotation. However, as Wave One files have their own unique reverse design, they can only be used with the WaveOne motor with its reverse reciprocating function. The Wave One technique involves the following stages:

1. Straight line access, accepted protocol

2. Wave one file selection

3. Single-file shaping

4.Copious irrigation with $5 \% \mathrm{NaOCl}$ and EDTA before, during and after single-file shaping.

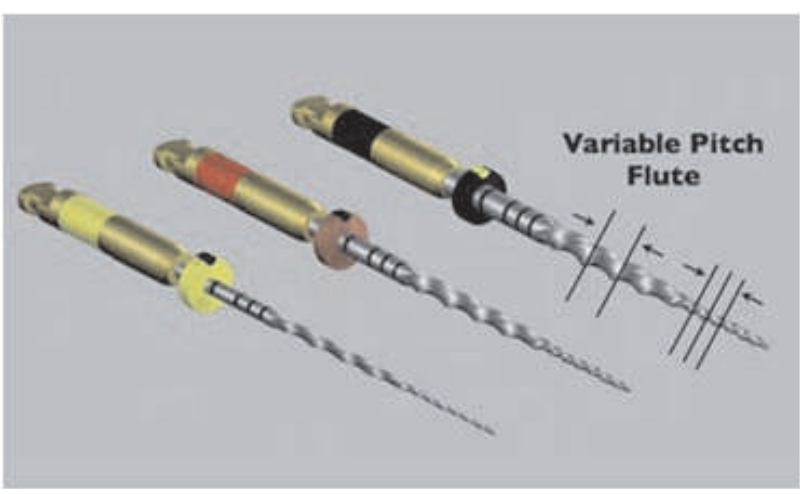

Figure 1: Wave One file
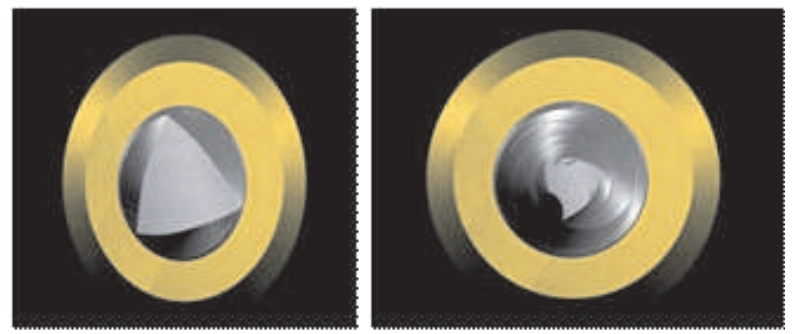

Figure 2: Convex triangular cross-section at the tip end and a convex triangular cross-section at the coronal end

Advantages of the WaveOne file reciprocating system ${ }^{8}$

1. Only one NiTi instrument per root canal and in most cases per tooth

2. Lower cost

3. Less instrument separation owing to the unique reciprocating movement that will prevent and/or delay the instrument advancing from plastic deformation to its plastic limit

4. Decreases global shaping time, allowing the clinician to spend more time cleaning the rootcanal System with enhanced irrigation techniques

5. Eliminates procedural errors by using a single Instrument rather than using multiple files

6. A new standard of care, eliminating the possibility of prior contamination owing to single use

7. Easy to learn

8. Easy to teac

\section{RECIPROC@ VDW}

A Specific Instrument Design an innovative instrument for a complete preparation RECIPROC ${ }^{\circledR}$ instruments have been designed with diameters and tapers which give an optimal apical preparation in most cases according to the canal anatomy, and using just one instrument. A regressive taper for a preparation with no unnecessary loss of tooth substance. RECIPROC ${ }^{\circledR}$ instruments are slimmer at 


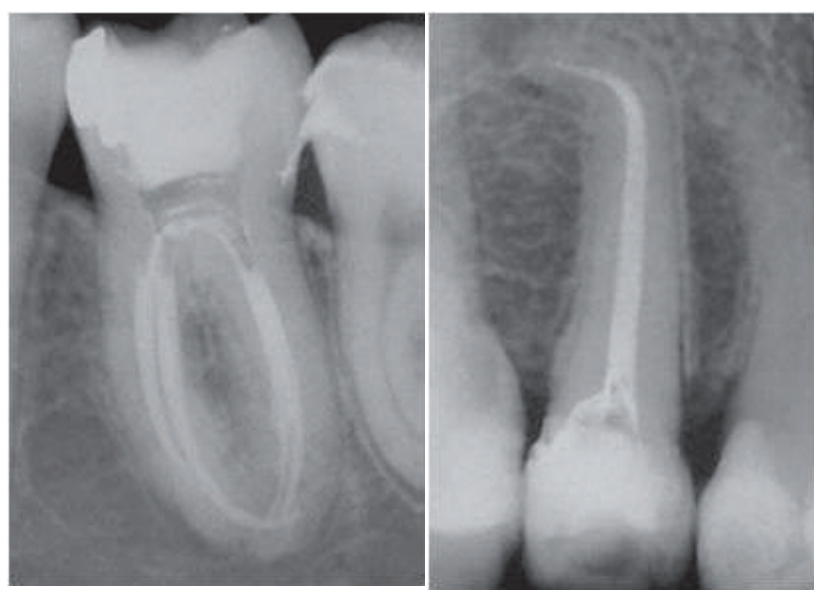

Figure 3: Severely curved and narrow canals can be successfully prepared with R25.This instrument is specifically designed for such canal anatomies. (Radiographs: Prof. Ghassan Yared, Ontario, Canada)

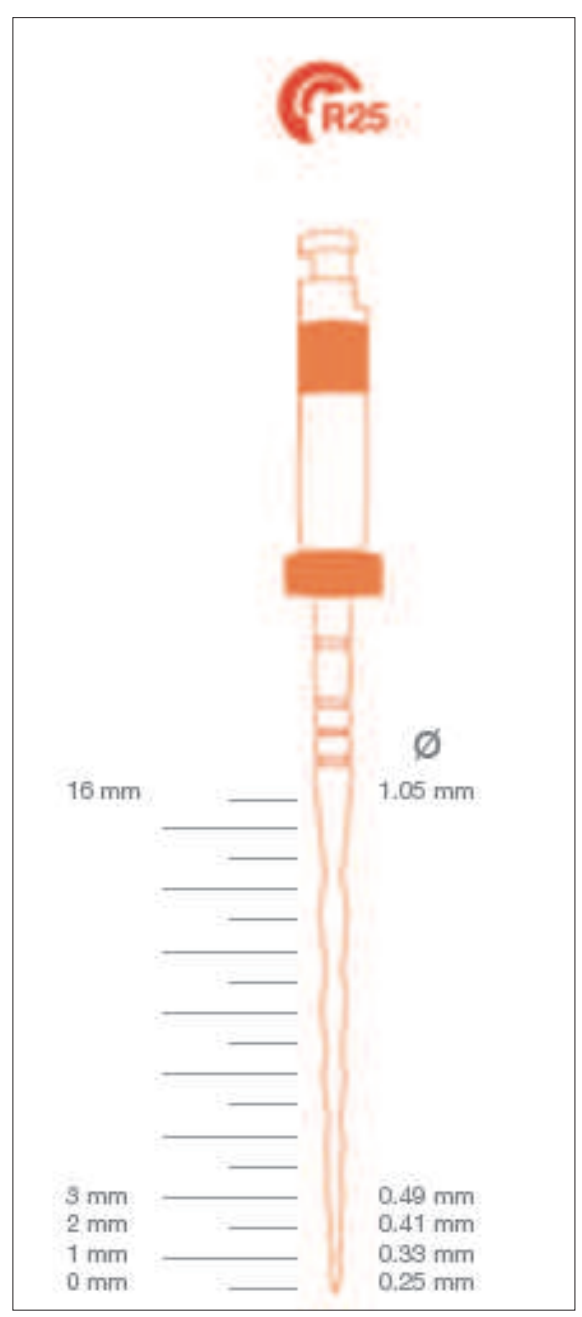

Figure 4 the end of the working part than most conical NiTi instruments of comparable diameter, preventing unnecessary loss of tooth substance in the coronal part. This system is suitable for preparation of severely curved and narrow canals Also severely curved and narrow canals can be prepared with one RECIPROC $®$ instrument (Figure 3 ). ${ }^{8}$

In reciprocation, clockwise and counterclockwise angles determine the amplitude of reciprocation, the right and left rotations. These angles, stored in the motor, are significantly lower than the angles at which the RECIPROC@ instrument would usually fracture (if bound). When a reciprocating instrument binds in the canal, it will not fracture because it will never rotate past its specific angle of fracture. Therefore, the creation of a glide path to minimize binding is not required for the RECIPROC ${ }^{\circledR}$ instruments. The reciprocation technique's centering ability together with the design of the RECIPROC® instrument and its increased cutting ability allows the RECIPROC ${ }^{\circledR}$ instrument to follow the existing and natural path of least resistance, which is the root canal (Figure 4).

\section{One Shape (Micro Méga, Besançon, France)}

In contrast to some other single file systems, the One Shape file is used in continuous rotation, as known from many former multiple file rotary NiTi systems for root canal preparation. The unique design of the One Shape instrument (Figure 5) incorporates a variety of different cross sections along the active length of the file, which offers an optimal and improved cutting action in three zones of the root canal. Each instrument has been electro-polished to enhance cutting efficiency. One Shape's flexibility and unique downward movement ensures a highly effective apical progression. Minimal fatigue along the length of the entire file virtually eliminates the risk of accidental instrument separation. The One Shape instrument is actually available with a safety tip in size 25 at the tip with a continuous 0.06 taper (Figure 6). It is delivered in a sterile blister, as done by many other manufacturers, and should be used for one tooth only and then discarded. It is designed for a maximum of three or four canals in one tooth and should not be sterilized, because the cutting efficiency decreases severely. Practitioners can use their existing endodontic motor with the One Shape instrument. One Shape is optimally designed to prepare the majority of root canal configurations. One Shape is one of the few single file instruments used in continuous clockwise rotation for a quick and probably safe root canal preparation (Figure 7). ${ }^{9}$ 


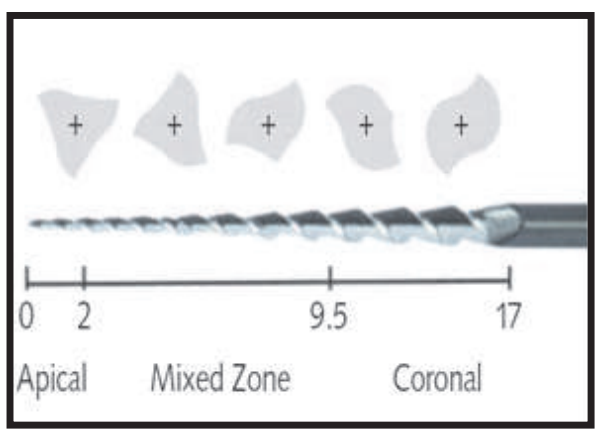

Figure 5: The unique design of the cross-section of the One Shape file. At the apical part there are three symmetrical cutting edges. In the middle the number decreases to two cutting edges; this part is asymmetrical. In the coronal part there are two Sshaped cutting edges.

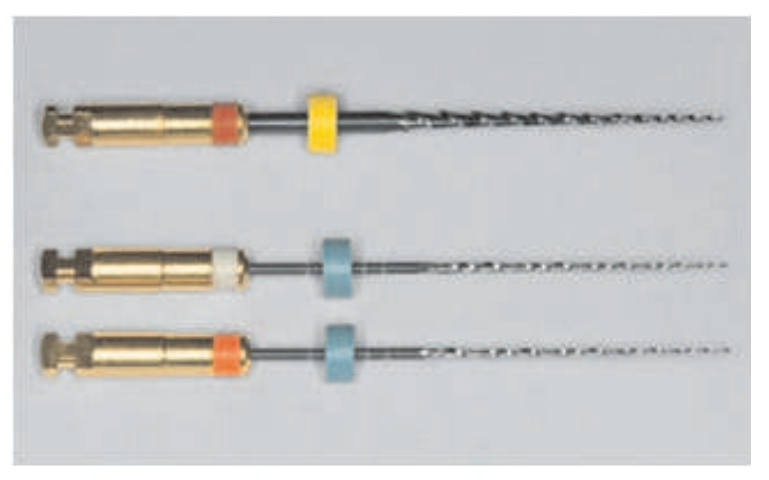

Figure 6: The single file system One Shape on top (Micro Méga, Besançon, France). The size at the tip is 25 with a taper of 0.06 . The working length is $17 \mathrm{~mm}$. The files are available in $21 \mathrm{~mm}$, $25 \mathrm{~mm}$ and $29 \mathrm{~mm}$. Glide path management can be performed using the recently introduced asymmetrical G-Files (G1 = size 12 and G2 = size 17, Micro Méga), the two files with the orange and white ring.

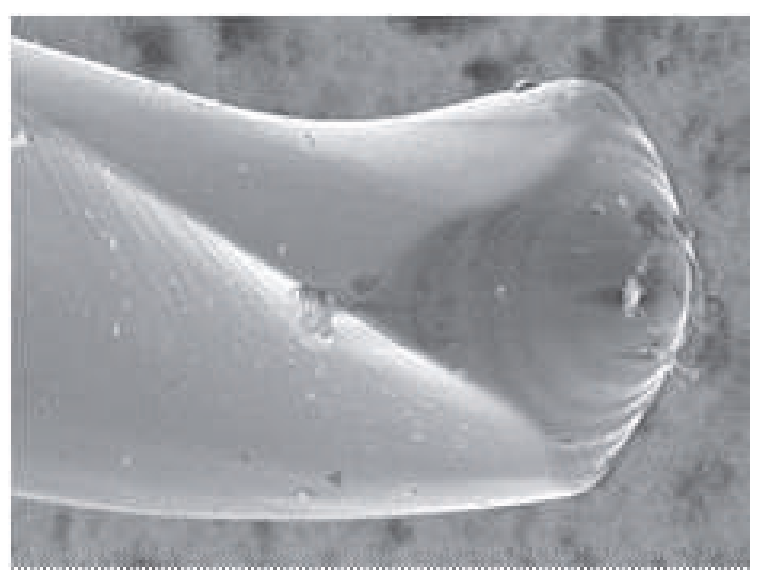

Figure 7: Safety tip
As recommended by the manufacturer, the rotational speed for One Shape is $400 \mathrm{rpm}$. The use of torquecontrol motors or torque-limited rotation handpieces has strict recommendations and torque should be set to $4 \mathrm{Ncm}$.

\section{KOMET F360 ${ }^{\circledR}$}

(Komet Brasseler, Lemgo, Germany)

F360 system of NiTi files with a taper of 04 for the mechanical preparation of root canals (Figure 8).

Torque $1.8 \mathrm{Ncm}$, Speed 250-350rpm, Max speed 500rpm

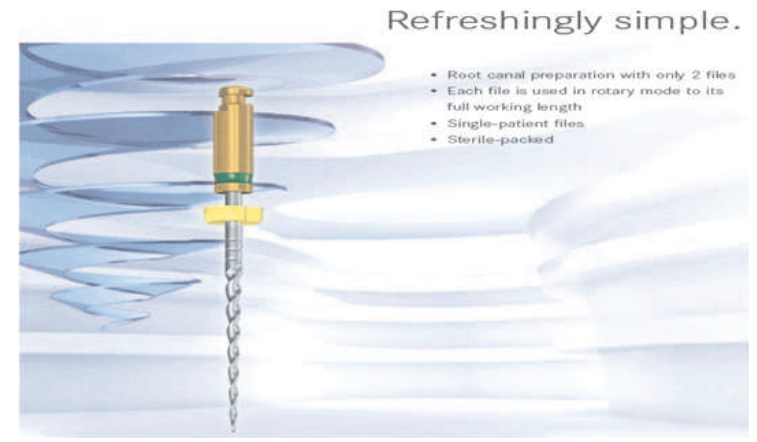

Figure 8

Indication: Rotary preparation of straight and curved root canals in a torque limited power system.

Contraindication: Use in root canals with pronounced abrupt curves in apical region and severely calcified root canals.

Clinical sequence/ appropriate use: The instrument is designed for single use i.e, on one patient only upto 5 root canals can be treated with each file.

Check instrument for plastic deformation, e.g. untwisted blades, in the region of the working part during the course of the treatment, during intermediate cleaning. Discard any imperfect files prior to the use of the F360 files.

\section{Method of Use of $\mathbf{f 3 6 0}$}

- Insert the file into canal in rotary mode

- Used in picking motion i.e, the files are moved up and down the canal wall during a period of maximum 5-8seconds, according to the singlelength principle.

- Clean the instrument periodically during the treatment, for example in the interim block. 
- Rinse the root canal thoroughly in order to flush out all debris.

- Storage of file- store at room temperature in a dark and dry place.

- do not use after the expiry date. ${ }^{10}$

Why You Should Use This File: ${ }^{10}$

1. Single file for RCT

2. Unique double $\mathrm{S}$ cross-section

3. Sharp cutting edge

4. Large chip space

5. Lower bending moment

6. Excellent cleaning

7. Time saving

8. No special motor required

9. Single cone obturation

10. More economical

\section{CONCLUSION}

The Single File System is an exciting new concept in the preparation of the root canal. Whilst other teaching advocates the use of multiple NiTi files of different diameter and taper to gradually enlarge the root canal. But with these single file system only one file is required to prepare the canal to an adequate size and taper, even in narrow and curved canals. However, along with this, there must be a caveat. These files only shape the canal, extremely quickly in many instances, but they do not clean the root canal. It is the duty of teachers, clinicians and manufacturers to emphasize the role and importance $\mathrm{o}$ irrigation as a major determinant of endodontic success. Once it is fully appreciated that shaping and cleaning the root-canal system are irrevocably intertwined, then endodontics will be easier for all and available to all, and with the advancement of single file in continuous motion too like komet $\mathrm{f} 60$ and OneShape.

\section{REFERENCES}

1. Anil Dhingra, Punit Srivastava, Dipti Chadda, Satyabrat Banerjee. Simplify your Endodontics with Single File Systems- Case Reports. Journal of Dental and Medical Sciences. 2013; 6(6):44-51.

2. Sjogren U, Figdor D, Persson S, Sundqvist G. Influence of infection at the time of root filling on the outcome of endodontic treatment of teeth with apical periodontitis. Int Endod J 1997; 30(5): 297-306.

3. Schilder H. Cleaning and shaping the root canal. Dent Clin Amer 1974; 18(2): 269-96.

4. West JD. Endodontic predictability- "Restore or remove: how do I choose?" In: Cohen M, ed. Interdisciplinary Treatment Planning: Principles, Design, Implementation. Quintessence Publishing Co., 2008:123-64.

5. Roane JB, Sabala CL, Duncanson MG. The "balanced force" concept for instrumentation of curved canals. J. Endod 1985; 11(5): 203-11.

6. Johnson E, Lloyd A, Kuttler S, Namerow K. Comparison between a novel nickel titanium alloy and 508 Nitinol on the cyclic fatigue life of Profile 25/.04 rotary instruments. J Endod 2008; 34(11): 1406-9.

7. Walia HM, Brantley WA, Gerstein H. An initial investigation on the bending and torsional properties of Nitinol root canal files. J Endod 1998; 14(7): 340-51.

8. Prof. Ghassan Yared Ontario, Canada. Reciproc http://www.vdw-dental.com.

9. Christian R. Gernhardt One Shape - a single file NiTi system for root canal instrumentation used in continuous rotation ENDO (Lond Engl) 2013;7(3):211-216.

10. KOMET f360 (Komet Brasseler, Lemgo, Germany) simpleand safe. http://www.kometdental.de.

Source of Support: Nil, Conflict of Interest: None Declared 